\title{
ACQUIRED PORTAL-PULMONARY VENOUS ANASTOMOSIS COMPLICATING PARTIAL OESOPHAGO-GASTRECTOMY IN A PATIENT WITH PORTAL HYPERTENSION
}

\author{
BY \\ C. R. B. BLACKBURN \\ From the Clinical Research Unit,* Royal Prince Alfred Hospital, Sydney, Australia
}

(RECEIVED FOR PUBLICATION SEPTEMBER 23, 1955)

The medical management of patients with portal venous hypertension and bleeding from oesophageal varices is limited to the control of bleeding by balloon tamponage, the restoration of circulating blood volume, the prevention and treatment of hepatic coma, and the prescription of a suitable diet. On the other hand various surgical procedures may be carried out depending on the cause of the portal hypertension, the site of obstruction to the blood flow, the anatomy of the portal venous bed, and the degree of hepatic decompensation.

The surgical management of patients with extrahepatic portal obstruction and cavernomatous transformation of the portal vein is not wholly successful, and opinions differ as to which operative procedure secures the best result. The majority of surgeons prefer either end-to-side anastomosis of the splenic vein to the left renal vein or partial gastro-oesophagectomy. In some patients a prior splenectomy or the presence of an unsuitable splenic vein precludes the formation of a satisfactory anastomosis, and resection of the varixbearing area is usually preferred to ligation or injection of varices.

Partial oesophago-gastrectomy, that is resection of the varix-bearing area, is a palliative operation since portal hypertension is not relieved and may be accentuated, and bleeding may recur. It can only be regarded as a last resort in a young and otherwise healthy patient who has extra-hepatic portal obstruction and a normal liver.

The liability to recurrent haemorrhage after oesophago-gastrectomy depends on several factors of which the following are important: (a) The extent of the gastrectomy in terms of the number of oxyntic cells remaining, since acid peptic digestion must play a role in the development of acute or chronic bleeding from oesophageal or gastric

\footnotetext{
- Supported in part by the National Health and Medical Research Council of Australia.
}

varices in many patients. Acid peptic juice is not 음 essential, since we have seen acute and chronic bleeding from oesophageal varices after total gastrectomy and partial oesophago-gastrectomy have been carried out. (b) The capacity of anatomotic channels outside the resection site to carry blood, since this must influence the height of the portal pressure and the development of new anastomoses in the operation site. Bleeding from varices situated outside the gastro-intestinal tract is very rare, and anastomotic channels in such areas are beneficial in terms of decompressing oesophago-gastro-intestinal varices.

Late complications of oesophago-gastrectomy are uncommon and are largely restricted to recurrent bleeding and dyspepsia of various types. The development of a lethal cardiac complication does not appear to have been recorded previously, and its occurrence seems sufficiently important to place it on record.

A patient is reported in whom an acquired anastomosis between the portal and pulmonary venous systems is considered to have played a significant role in her death from cardiac failure after bleeding from intestinal varices. She died 11 years after splenectomy and three years after partial oesophago-gastrectomy was carried out as treatment for oesophageal varices and extrahepatic portal hypertension.

\section{Case RePort (P1525)}

Miss S., a school teacher with extra-hepatic portal venous obstruction, was admitted to the Royal Prince $\overparen{\Phi}$ Alfred Hospital 11 times between 1948 and 1953 for $\stackrel{\mathcal{P}}{?}$ the treatment of portal hypertension and its conse- 0 quences, and died in 1953 at the age of 19 years.

She was the elder of two girls; her mother and father were alive and well, and there was no relevant family history.

A few days after birth she was said to have vomited a small quantity of blood, and when 3 weeks of age 
to have become jaundiced for several days, but there was no history of umbilical sepsis.

She was well until the age of 6 years, when she was found to be anaemic and, shortly afterwards, had her first haematemesis. At 7 years of age she was found to have splenomegaly, a diagnosis of "Banti's syndrome" was made, and splenectomy was carried out the following year. After this operation she was well and led a normal existence till she was 14 years of age, when further gastro-intestinal bleeding occurred and she received her first blood transfusion. After this she had a series of severe haemorrhages which occurred at approximately six-monthly intervals, and she required blood transfusions for each of them.

In June, 1950, at the age of 16 years, she vomited 6 litres of blood over a period of 48 hours, and bleeding was controlled by oesophago-gastric balloon tamponage.

In August, 1950, she had a further massive haematemesis, and partial gastrectomy and excision of the lowest two inches of oesophagus with oesophagojejunostomy and jejuno-junostomy (Roux-en-Y) was performed by Mr. F. W. Niesche. The portal venous pressure, measured in a left gastro-epiploic vein, was $40 \mathrm{~cm}$. of saline. Dense adhesions to the splenic bed were noted.

Seven months after this operation she had another haematemesis, was given 4.5 litres of blood by transfusion, and oesophageal varices were demonstrated by radiography during a barium swallow. During the next eight months she received 15.5 litres of blood by transfusion, and it was decided to operate again as the varices appeared to be confined to the lower one-third of the oesophagus.

The oesophago-jejunostomy was explored transthoracically in October, 1951, but the operation was limited to the freeing of dense adhesions and the division of several large veins, as her condition deteriorated rapidly.

Six months later, in April, 1952, she had a small haematemesis, and three weeks later the oesophageal varices were injected with 7-8 ml. of $10 \%$ sodium morrhuate through an oesophagoscope. Oesophagoscopies in September, 1952, and March, 1953, and radiographs of a barium swallow all failed to reveal any varices. The results of liver function tests were : thymol turbidity 8 units (normal up to 12), zinc sulphate turbidity 7 units (normal up to 12), total serum protein concentration $6.1 \mathrm{~g} . \%$, serum albumin $4.1 \mathrm{~g} . \%$, serum bilirubin $0.2 \mathrm{mg} . \%$.

In late 1952 there was definite cardiac enlargement involving the left ventricle, and this was especially apparent when radiographs of the heart taken in October, 1951, were compared with those taken in September, 1952 (Fig. 1).

The last 12 months of her life were characterized by a chronic hypochromic anaemia and occult blood in the stools continuously, but she continued to work as a kindergarten teacher.

In October, 1953, she was readmitted, having haematemeses and melaenas and, despite transfusions of blood, continued to bleed rapidly, became restless, stuporose, pale, and cold. She was resuscitated, but on the second day a 90 -minute period of rapid blood transfusion was followed by the development of early pulmonary oedema, pulsus paradoxus was observed, and an electrocardiogram showed a bizarre tracing interpreted as supraventricular tachycardia. Despite the administration of quinidine and procaine amide intravenously and measures designed to combat pulmonary oedema the tachycardia persisted at 100 to 180 beats per minute, and she died of cardiac failure two days after admission.
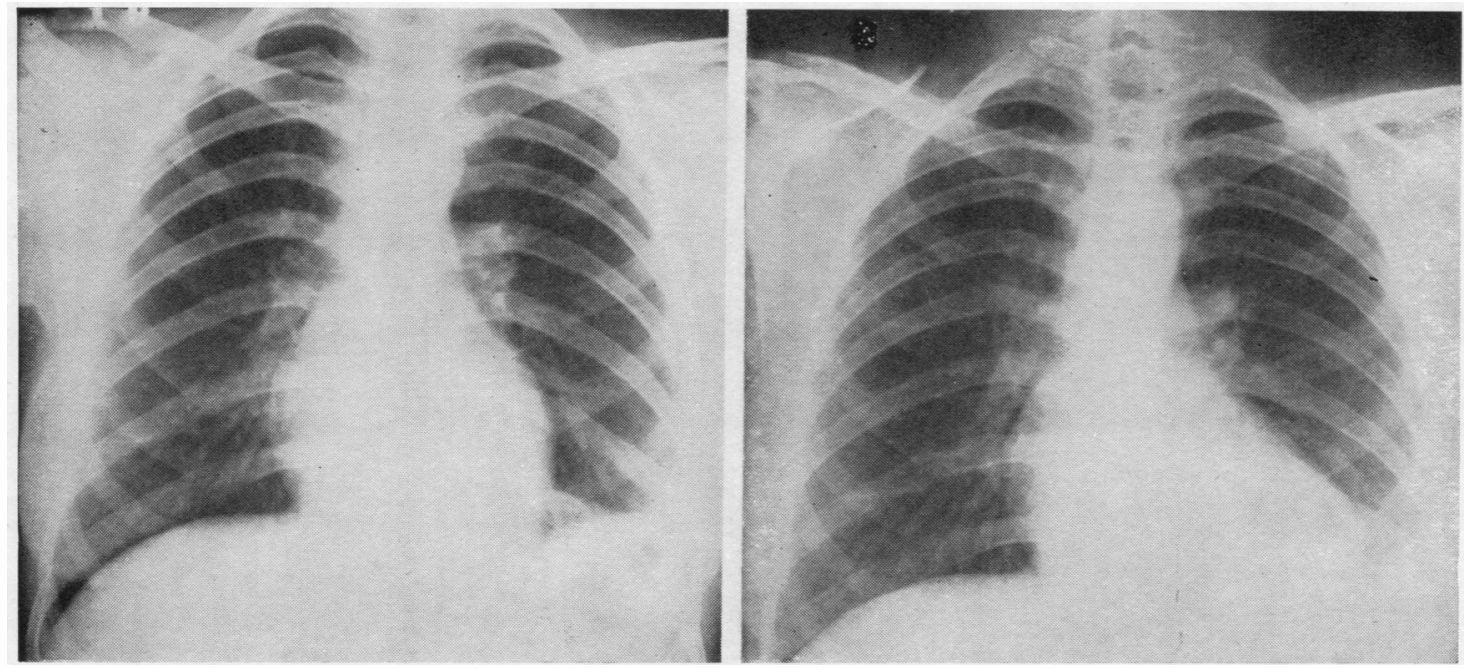

FIG. 1.-Enlargement of the left ventricle after the second transthoracic exploration of the cardio-oesophageal region (left film taken before second exploration, right film 11 months later). 


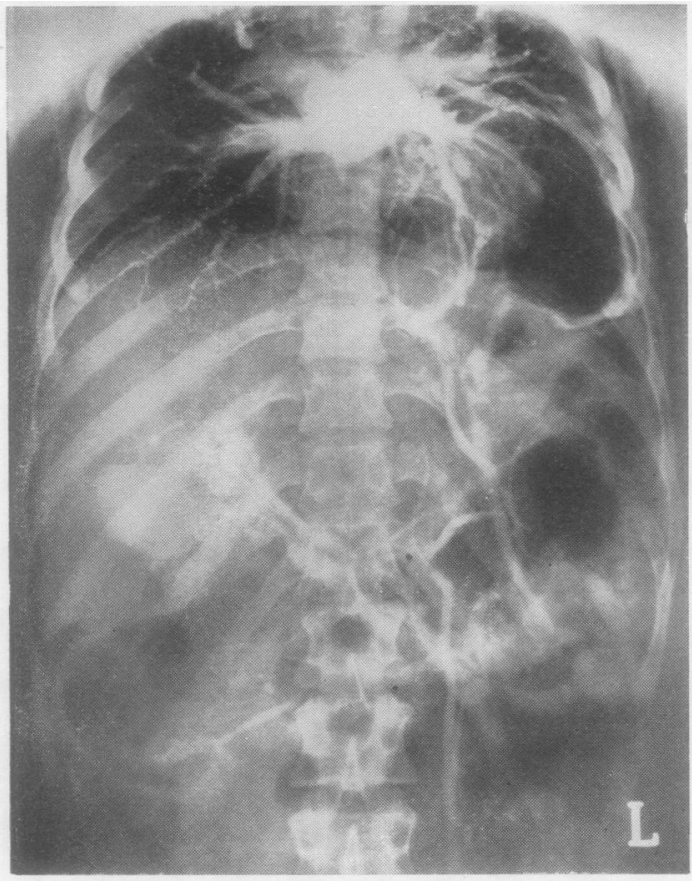

FIG. 2.-Post-mortem radiograph of the thorax and upper abdomen taken after injecting a barium sulphate suspension into the superior mesenteric vein.

\section{NECROPSY}

A necropsy was performed three hours after death.

Radiographic Findings. - The abdomen was opened and a barium sulphate suspension was injected into the superior mesenteric vein. Radiographs of the thorax and upper abdomen demonstrated large venous anastomoses between the portal venous bed and the thoracic cage and left pulmonary veins, so that the barium suspension filled the left auricle and the pulmonary venous bed. There was obstruction to the portal vein which extended into the porta hepatis but ended in a varicose stur.p without branches (Fig. 2).

Anatomical Findings.-The abnormal findings were confined to the thoraco-abdominal viscera, which were removed in one block and dissected later.

Venous System.-There was a cavernomatous transformation of the portal vein beginning at its formation at the head of the pancreas and extending well into the porta hepatis (Fig. 3) but not out into the liver substance. The anatomy of the portal vein corresponded with that depicted in the radiograph shown in Fig. 2. All the veins normally leading into the portal vein were grossly dilated, and especially those running in the pancreas into the left adrenal and those running into the anastomotic jejunal loop. There was a large varix on the anti-mesenteric surface of the jejunal loop in the thorax, and this was the site of an anastomosis between the superior mesenteric venous

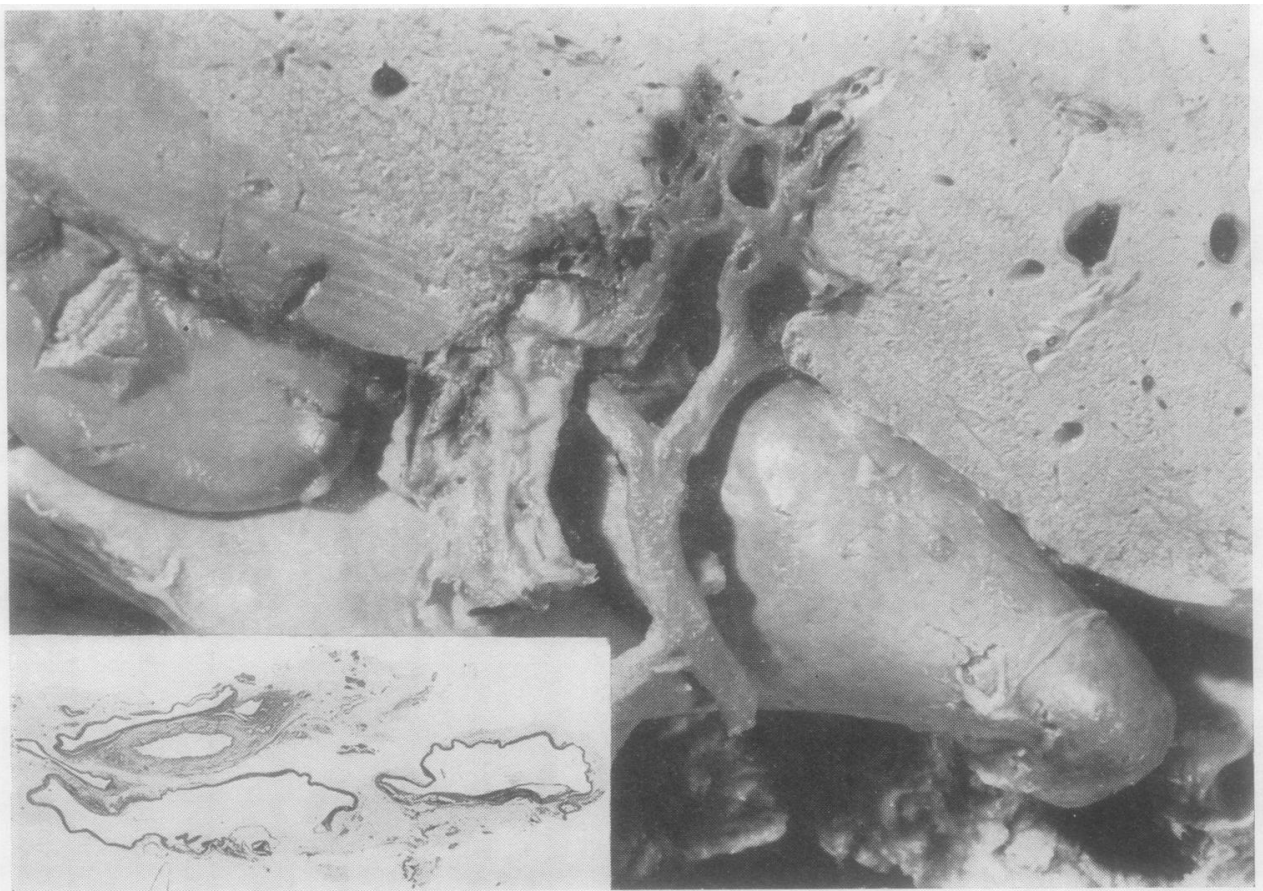

FIG, 3.-Dissected porta hepatis showing a cavernomatous portal vein surrounding the bile duct. Insert is a low-power view of a transverse section of the portal vein and bile duct. 


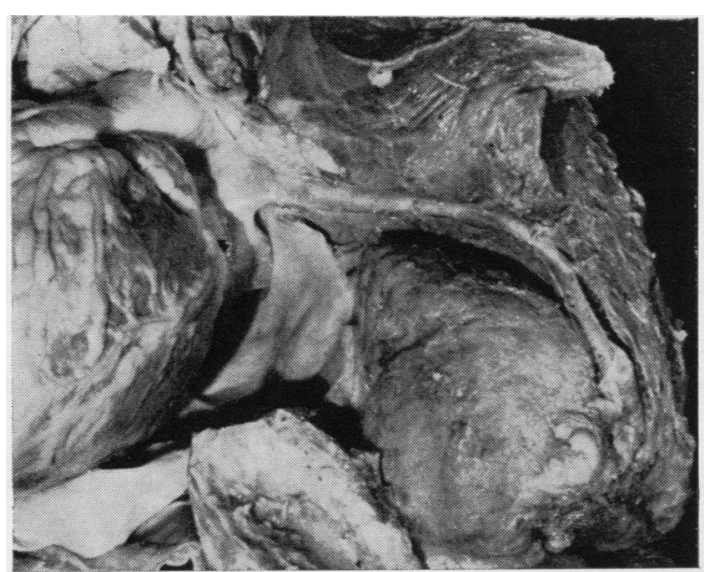

FIG. 4.-The varix on the jejunum communicates with a large vein running along the border of the left lung and enters the left pulmonary vein as it enters the left auricle.

system and a large subpleural left pulmonary vein which ran along the medial border of the lower lobe of the left lung. The dilated pulmonary vein entered a large left pulmonary vein just before it entered the left atrium (Fig. 4), and its minimum width after formalin preservation was $3 \mathrm{~mm}$. There was another smaller varix on the postero-medial surface of the thoracic jejunal loop which also connected with the pulmonary veins. The azygos venous system was grossly dilated. Small oesophageal varices could be demonstrated after section of the oesophagus, but a perforated varix in the jejunum $2 \mathrm{~cm}$. below the oesophageal anastomosis appeared to be the cause of the final bleeding (Fig. 5a). Varices could be demonstrated running across the line of anastomosis of the jejunum to the oesophagus (Fig. 5b). There were

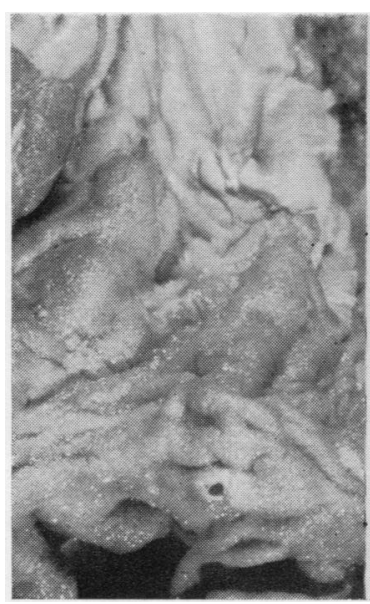

(5a)

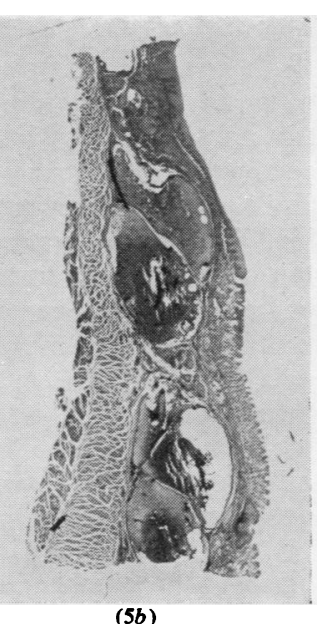

(5b)
FIG. 5a.-The oesophago-jejuna anastomosis with a perforated varix in the lower part of the photograph.

FIG. 5b.-Vertical section through the oesophago-jejunal anastomosis showing a continuous series of varices. dense adhesions between the liver, diaphragm, jejunum, lung, and pericardium.

Oesophagus, Small Intestine, and Stomach.-The oesophageal mucosa appeared normal. No ulcers were demonstrated and the anastomoses were patent and quite satisfactory. There was a moderate amount of blood in the intestinal tract, but there was only a little immediately below the ruptured varix.

Heart.-The heart weighed $250 \mathrm{~g}$., but there was gross hypertrophy of the left ventricle, the wall of which was $25 \mathrm{~mm}$. thick whilst the right ventricular wall was $4 \mathrm{~mm}$. thick (Fig. 6). There were no abnormalities of the valves or coronary arteries; the aorta was normal. There were no abnormal findings on microscopical examination of the heart.

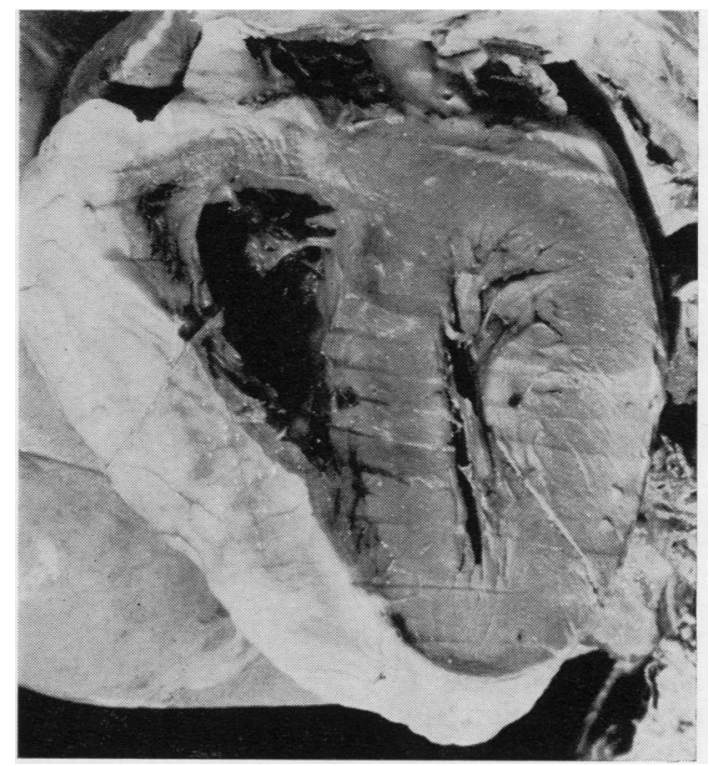

Fig. 6.-Heart cut open to show the enormous hypertrophy of the left ventricle.

Liver.-The architecture of the liver was normal macroscopically and microscopically except that the portal veins appeared smaller than normal.

Lungs. - There was some oedema, but there were no vascular changes suggesting pulmonary hypertension.

\section{Discussion}

The history and course of this patient's illness were, at first sight, not unusual. She developed portal obstruction at an early age, perhaps in the immediate neonatal period, and portal hypertension became clinically important in childhood when she bled from oesophageal varices. Recurrent bleeding made surgical measures essential and splenectomy was carried out at the age of 8 years. Bleeding continued, and, despite partial oesophagogastrectomy, re-exploration and ligation of veins 
near the oesophago-jejunostomy site, and injection of oesophageal varices, she died from a ruptured jejunal varix.

Necropsy revealed cavernomatous transformation of the portal vein, but the changes did not extend far into the liver. The liver was normal both on macroscopical and microscopical examination.

The final bleeding site was in the jejunal mucosa below the oesophago-jejunal anastomosis, and it led directly into a large varix in the submucosal venous plexus. Peptic digestion could not have played a major role in the rupture of this varix because the oesophagus did not contain gastric mucosa and a Roux-en-Y anastomosis had been carried out.

Large acquired anastomotic channels between the portal venous bed and the left pulmonary veins, via the superior mesenteric vein, were of considerable interest and must have been the cause of the left ventricular enlargement, as no other cause was found. The radiological examination at necropsy demonstrated the functional significance of the anastomoses, since the bulk of the opaque medium flowed into the pulmonary veins and left atrium, though the site of the injection was a mesenteric vein well removed from the site of anastomosis.

The final episode in this patient is considered to have resulted from the following sequence of events. Considerable blood loss caused a gross reduction of left ventricular inflow indirectly from splanchnic vasoconstriction and directly from decreased flow through the portal-pulmonary anastomoses. She was given too much blood by rapid transfusion, and the increased blood volume, especially in the splanchnic bed, increased the load on the left ventricle both from the lungs and through the anastomoses. She developed left ventricular failure with pulmonary oedema and uncontrollable tachycardia and this finally caused her death. The pulsus paradoxus is difficult to explain, but it may be suggested that it was related to increased venous return to the failing left ventricle which resulted from the combined effect of increased intra-abdominal pressure and decreased intra-thoracic pressure during inspiration acting on the blood flow through the portalpulmonary anastomoses. The load exceeded the left ventricular capacity to respond and cardiac output decreased.

Whereas congenital communications between the portal and pulmonary veins are well recognized, the occurrence of functionally significant acquired anastomoses between the left pulmonary veins and the portal venous system is most unusual. Con- genital communications may drain the venous $\stackrel{\overrightarrow{\vec{P}}}{\rightarrow}$ blood from one or both lungs into the portal vein itself, the ductus venosus, or the left gastric vein, $\frac{\partial}{=}$ and in these circumstances the left ventricle is $\frac{\bar{\sigma}}{\overrightarrow{ }}$ small and the condition is most often found in $\stackrel{\varnothing}{\triangle}$ infants dying during the first week of life (Edwards, के 1953). Partial connexions running in the oeso-

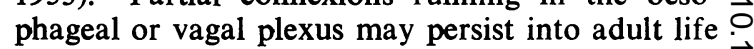
(Butler, 1952). Acquired anastomoses develop in $\overrightarrow{\vec{\omega}}$ the same manner as those that are sought by ${ }_{\infty}$ carrying out the Talma-Morison operation in $\overrightarrow{\vec{x}}$ patients with portal hypertension. It is assumed that the high portal pressure played a major role $\overrightarrow{\vec{\omega}}$ in our patient and that the division of other $\vec{\omega}$ anastomotic channels during the two operations in the region of the diaphragm tended further to $\frac{}{\partial}$ raise the portal pressure. The second radiograph $\vec{r}$ of the chest has shadows that are consistent with the outline of the large portal-pulmonary anasto- $\frac{\bar{S}}{\mathcal{S}}$ mosis demonstrated at necropsy, and this was un- $\vec{\theta}$ disturbed by the second operation. Furthermore, $\mathcal{C}$ the enlargement of the left ventricle occurred after the second operation which must have directed still more portal blood to the left auricle and ventricle.

Acquired anastomotic venous channels in patients with portal hypertension convey blood $\frac{\%}{\varnothing}$ from the high pressure portal system, 25-30 $\mathrm{mm}$. $\stackrel{\unrhd}{\square}$ $\mathrm{Hg}$, to the lower pressure pulmonary veins, 2-3 $\overrightarrow{\mathrm{O}}$ $\mathrm{mm} . \mathrm{Hg}$. There is a greater output by the left 3 ventricle than the right, so that left ventricular hypertrophy and cardiac failure may ultimately? develop. These changes are in sharp contrast to those in patients with congenital anastomoses and, 음 in some regards, they resemble those associated $\stackrel{x}{x}$ with a patent ductus arteriosus.

The possible development of anastomoses between the portal and pulmonary veins is an impor- $\frac{0}{3}$ tant consideration when recommending oesophagogastrectomy for patients with portal hypertension? and bleeding oesophageal varices. Recurrent $\frac{D}{5}$ bleeding has been stressed as the major risk after this operation, but radiographic and other evidence $N$ of cardiac enlargement, especially of the left $t_{N}$ ventricle, should make one suspect changes such as are reported here. The danger of myocardial 6 insufficiency would be accentuated in those patients? with cirrhosis of the liver who have a high cardiace output. It seems desirable to avoid intra-thoraciced anastomoses joining the oesophagus to the jejunum ${ }^{+}$ or to the stomach, since their venous drainage is close to the blocked portal vein. An argumentक can be put forward to support the claims of oeso- $\frac{}{P}$ phago-gastrectomy with colonic replacement, sinced the inferior mesenteric venous system appears les often to be involved in the formation of significants 
varices. Finally an abdominal approach may be better than a thoracic one.

The rapid increase in size of the left ventricle after a second exploration of the oesophageal region emphasizes the danger of dividing beneficial anastomoses in patients with portal hypertension. When this is done still more blood flows through the remaining varicose channels and increases the liability to haemorrhage. In our patient this increase in flow contributed to her death from cardiac failure.

In Fig. $5 b$ it can be seen that dilated venous channels crossed over the line of anastomosis between the oesophagus and jejunum, and the channels were very extensive and thin walled. This finding provides good evidence that the view that limited cuff resections of stomach and oesophagus may be effective for the treatment of oesophageal varices is not well founded. This view is based, in part at least, on the erroneous hypothesis that varicosities only develop in preformed chan- nels and not in acquired anastomoses and so would not form across a line of gastric or jejunal anastomosis to the oesophagus.

\section{SUMMARY}

A patient with extra-hepatic portal hypertension is reported in whom splenectomy and then partial oesophago-gastrectomy were performed for the relief of bleeding oesophageal varices. The development of an acquired portal pulmonary venous anastomosis led to left ventricular enlargement and ultimately contributed to her death from cardiac failure at the age of 19 years. Her final bleeding episode resulted from rupture of jejunal varices. The significance of this type of acquired veno-venous anastomosis is discussed in relation to the operative treatment of portal hypertension by oesophago-gastric resection.

\section{REFERENCES}

Butler, H. (1952). Thorax, 7, 249.

Edwards, J. E. (1953). Proc. Mayo Clin., 28, 441. 